\title{
INFESTAÇÃO DE VETORES DA TRIPANOSOMÍASE AMERICANA NO AMBIENTE DOMICILIAR, NO ESTADO DE GOIÁS.
}

Ionizete Garcia da Silva*, João Leite da Silva**, Marlene de Fátima Camargo*, Carmeci Natalina Elias*, Adelair Helena dos Santos*, Heloisa Helena Garcia da Silva*, Eliana Isac*, Rosa de Belém das Neves Alves*.

\section{RESUMO}

Foram investigados domicílios e anexos em 161 municipios do Estado de Goiás e capturaram-se 13.951 triatomineos. As espécies de triatomíneos encontram-se na ordem decrescente de suas frequiências de infestação domiciliar: Triatoma sordida $-87,11 \%$, Rhodnius neglectus $-6,01 \%, T$. infestans - 3,86\%, Panstrongylus megistus $-2,59 \%$, T. pseudomaculata $-0,24 \%, P$. diasi $-0,12 \%, T$. costalimai $-0,08 \%, P$. geniculatus $-0,04 \%, T$. lenti $-0,03 \%$. O indice de infecção natural do triatomineo pelo Trypanosoma cruzi, foi encontrado apenas nas 5 primeiras espécies, consideradas de hábitos sinantrópicos, em ordem decrescente: $T$. sordida $-0,37 \%, T$. infestans $-0,11 \%, P$. megistus $-0,07 \%, T$. pseudomaculata $-0,02 \%$ e $R$. neglectus $-0,01 \%$.

UNITERMOS: Tripanosomíase americana. Trypanosoma cruzi. Infestação. Triatomíneos. Vetores.

\section{INTRODUÇÃO}

O conhecimento dos índices de infestação e infecção de triatomíneos no ambiente domiciliar é fundamental e indispensável para o planejamento das ações de controle $^{l} 24 \quad 4 \quad 5$. A atualização de dados é imprescindivel para a avaliação de campanhas antitriatomínicas em andamento ou as projetadas para execução. É útil também para avaliar a freqüência com que as espécies domiciliadas chegam ao ambiente do homem, assim como, o grau de adaptação destas ao ecótopo artificial, possibilitando desta forma, estimar o risco da transmissão vetorial e visualizar, numa dimensão atual, o problema da tripanosomíase americana, no Estado de Goiás.

\footnotetext{
Laboratório de Entomologia, convênio UFG/Fundação Nacional de Saúde -Goiânia-Goiás, C.P.131.

** Fundação Nacional da Saúde - Goiânia/GO.

Recebido para publicação em 31/05/95
} 
SILVA, I.G.; SILVA, J.L.; CAMARGO, M.F.; ELIAS, C.N.; SANTOS, A.H.; SILVA, H.H.G.; ISAC, E.; ALVES, R.B. N. Infestação de vetores da tripanosomiase americana no ambiente domiciliar, no Estado de Goiás. Rev. Pat. Trop. 24 (1): $41-47$, jan/jun. 1995

\section{MATERIAL E MÉTODOS}

Foi realizado, no período de 1992/93, em Goiás, através de investigação ativa no domicílio e anexos, um levantamento de triatomíneos para se conhecer os indices de infestação e de infecção pelo Trypanosoma cruzi. Os triatomíneos indices de infestação e de infecção pelo Trypanosoma polietileno e remetidos para o laboratório para identificação das espécies e deteç̧ão de tripanosoma. Foram laboratório para identificação das expécies direta ${ }^{13}$. Para capturados 13.951 triatomineos e examinat cada triatomíneo positivo foram feitas tres fecal com $T$. cruzi, misturados com solução fisiologica, na proporção de $5 \mathrm{ml}$ desta solução para $1 \mathrm{ml}$ de soro humano. Retiravam-se duas gotas dessa solução e colocava-se em lâminas limpas e fixavam com álcool metílico a $96 \%$. Após a secagem, a lâmina era imersa no azul de metileno, posteriormente lavada em água destilada e corada pelo Giemsa durante 30 minutos.

\section{RESULTADOS E DISCUSSÃO}

As espécies de triatomíneos encontram-se na ordem decrescente de suas freqüências de infestação domiciliar: Triatoma sordida - 87,11\%, Rhodnius freqüências de infestação domicilar. Triatoma $-3,86 \%$, Panstrongylus megistus - 2,59\%, T neglectus - 6,01\%, $T$, $P$. diasi - 0,12\%, T costalimai - 0,08\%, P. geniculatus pseudomaculata - $0,24 \%, P$. dia $0,04 \%, T$. lenti $-0,03 \%$. A infecção natural do triatomíneo pelo Trypanosoma cruz foi encontrada apenas nas 5 primeiras espécies, consideradas de hábito sinantrópicos: $T$. sordida $-0,37 \%, T$. infestans $-0,11 \%, P$. megistus $-0,07 \%, T$ pseudomaculata $-0,02 \%$ e $R$. neglectus - $0,01 \%$
SILVA, I.G.; SILVA, J.L.; CAMARGO, M.F.; ELIAS, C.N.; SANTOS, A.H.; SILVA, H.H.G.; ISAC, B.; ALVES, R.B.N. Infestação de vetores da tripanosomíase americana no ambiente domiciliar, no Estado de Goiás. Rev. Pat. Trop.24 (1): 41 - 47, jan/jun. 1995

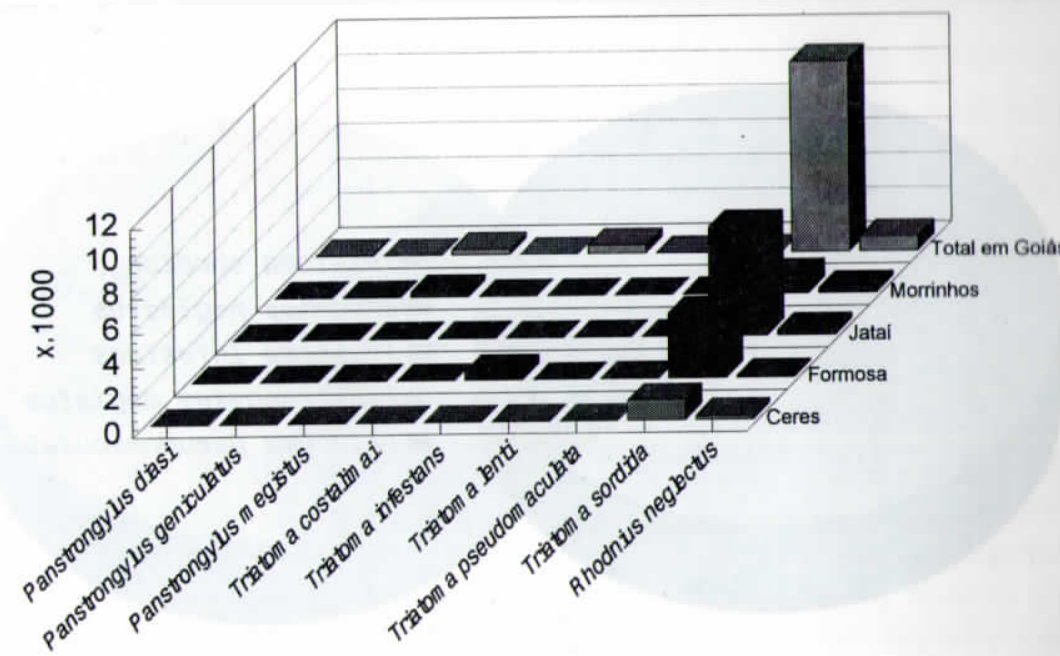

Fig.1 Frequência de triatomíneos capturados no ambiente domiciliar no estado de Goiás, agrupados por distritos, no período de 1991/92.

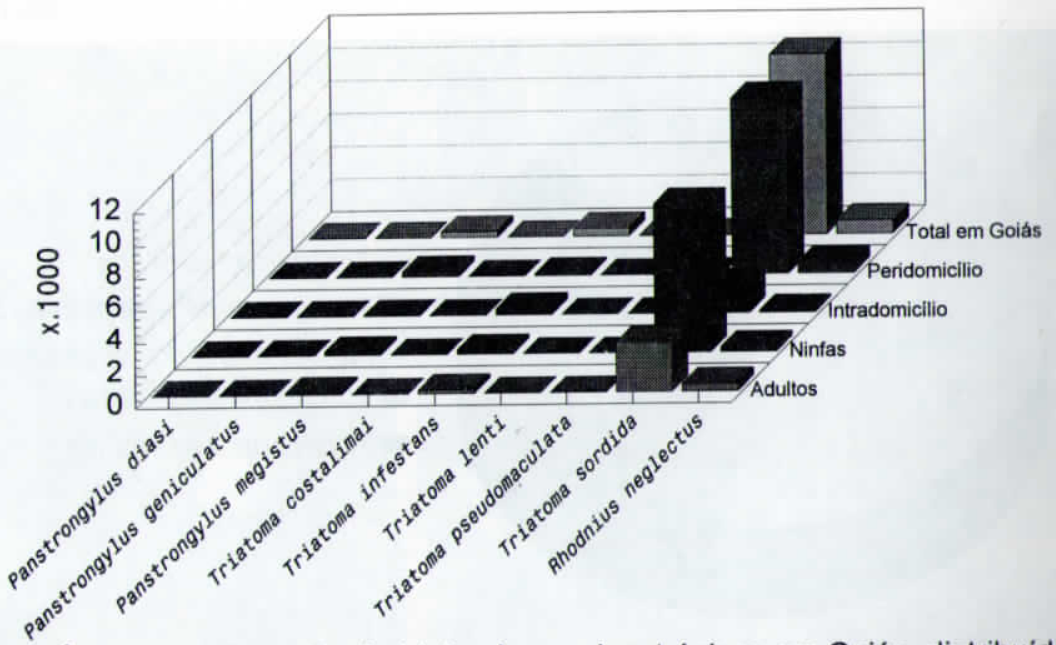

Fig. 2 Índice de infestação de triatomíneos sinantrópicos em Goiás, distribuidos de acordo com o local de captura, no período de 1991/92. 
SILVA, I.G.; SILVA, J.L.; CAMARGO, M.F.; ELIAS, C.N.; SANTOS, A.H.; SILVA, H.H.G.; ISAC, E.; ALVES, R.B.N. Infestaçăo de vetores da tripanosomf́ase americana no ambiente domiciliar, nœ Estado de Goiás. Rev. Pat. Trop.24 ( 1 ): 41 - 47, jan/jun. 1995

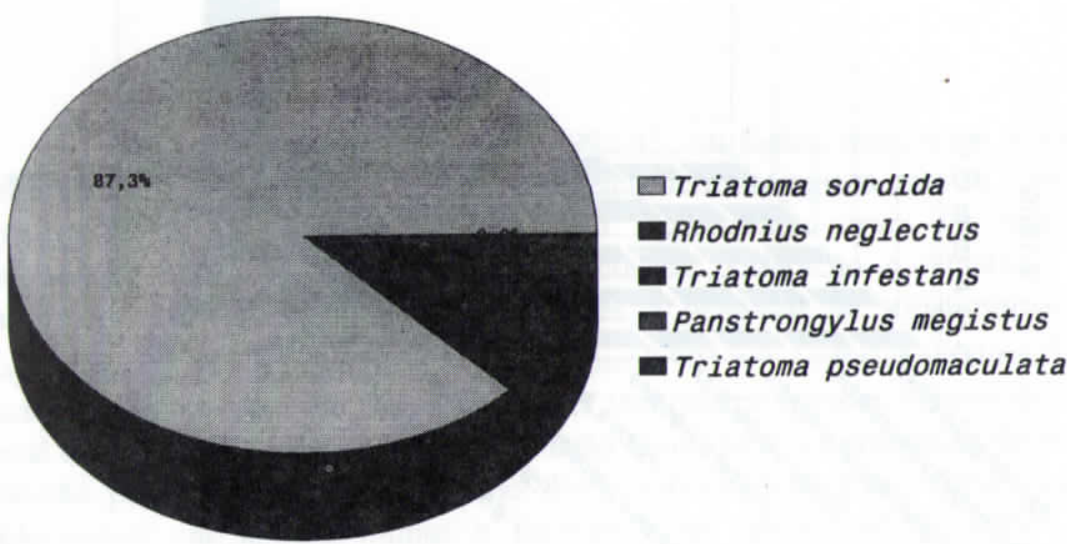

Fig.3 Índice de infestaçāo de triatomíneos sinantrópicos no estado de Goiás, no período de 1991/92.

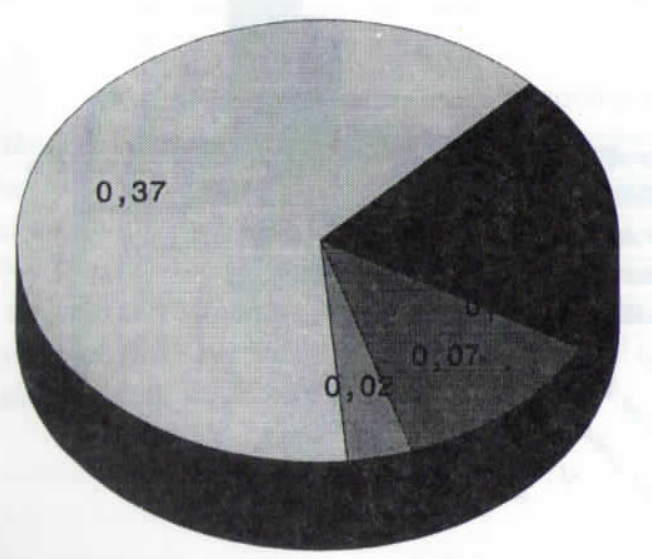

$\square$ Triatoma sordida

- Triatoma pseudomaculata

- Panstrongylus megistus

- Rhodnius neglectus

- Triatoma infestans

Fig.4 Índice de infecçāo $(\%)$ das espécies sinantrópicas de triatomineos pelo Trypanosoma cruzi, em Goiás, no periodo de $1991 / 92$.
SILVA, I.G.; SILVA, J. ; CAMARGO, M.F.; ELIAS, C.N.; SANTOS, A.H.; SILVA, H.H.G.; ISAC, E.; ALVES, R B N Infestaço de vetores da tripanosomíase americana no ambiente domiciliar, no Estado de Goiás. Rev. Pat. Trop.24 ( 1 ): 41 - 47, jan/jun. 1995

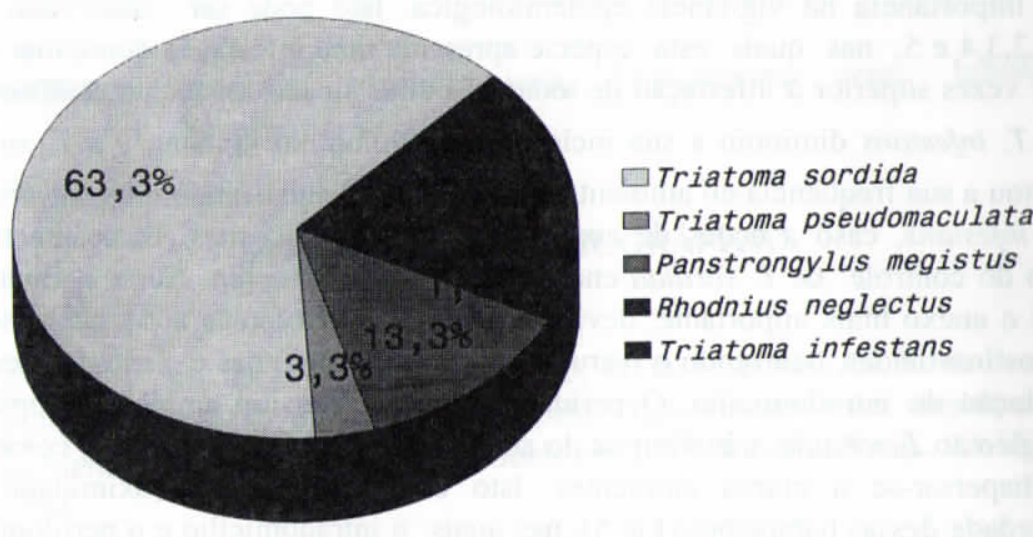

Fig.5 Distribuição da positividade entre as espécies sinantrópicas Fig.5 Distribuiçấo da $1991 / 92$.

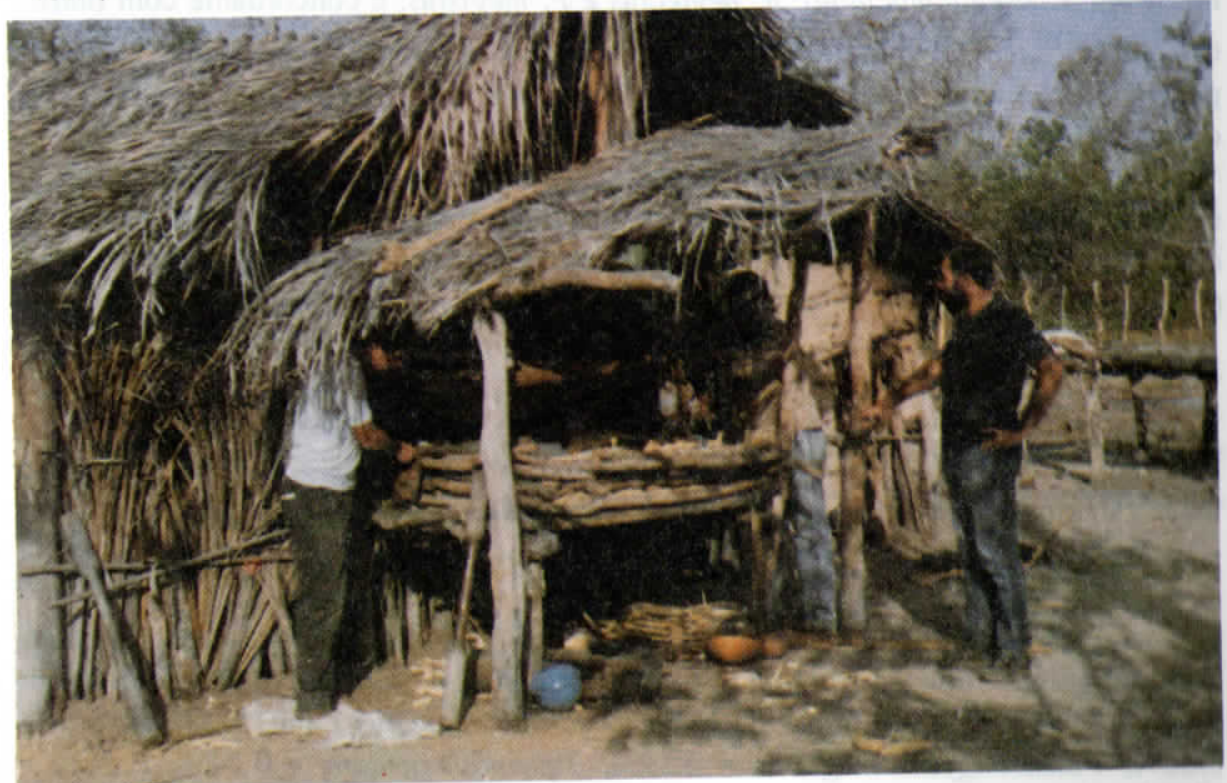

Fig.6 Habitação rural infestada por triatomíneos, localizada no município de Alvorada do Norte. 
SILVA, I.G.; SILVA, J.L.; CAMARGO, M.F.; ELIAS, C.N.; SANTOS, A.H.; SILVA, H.H.G.; ISAC, E.; ALVES, R.B.N. Infestação de vetores da tripanosomíase americana no ambiente domiciliar, no Estado de Goiás. Rev. Pat. Trop.24 ( 1 ): $41-47$, jan/jun. 1995

Entre essas espécies sinantrópicas, $T$. sordida tem-se mostrado como a de maior importância na vigilância epidemiológica. Isto pode ser observado nas Figs.1,2,3,4 e 5, nas quais esta espécie apresenta uma infestação domiciliar cerca de três vezes superior à infestação de todas as outras sinantrópicas juntas. Enquanto que o $T$. infestans diminuiu a sua incidência no domicílio humano ${ }^{1}$, o $T$. sordida aumentou a sua freqüência no ambiente domiciliar ${ }^{145}$ sinalizando uma substituição do $T$. infestans, caso a ações de controle não sejam eficientes. Basicamente , o desafio do controle de $T$. sordida encontra-se no peridomicílio. Nesse ambiente, o paiol é o anexo mais importante, devido à proteção que oferece aos triatomíneos à ação dos inseticidas, ocorrendo o recrudescimento das colônias e, posteriormente, a reinfestação do intradomicílio. O peridomicílio tem sido um ambiente propício e estratégico ao $T$. sordida, transferir-se do ambiente silvestre ao domiciliar, colonizarse e dispersar-se a outros ambientes. Isto ocorre devido à proximidade e à precariedade dessas habitações (Fig.5), nas quais o intradomicílio e o peridomicílio compartilham o mesmo ambiente, que são separados apenas por uma parede contígua. Esta situação é extremamente favorável à transferência do triatomíneo do ambiente considerado peridomicílio ao intradomicílio. A presença dominante de $T$. sordida no ambiente domiciliar, em relação às outras espécies sinantrópicas, $T$. infestans, T. pseudomaculata, $R$. neglectus e P. megistus, é concordante com outros trabalhos 145 . Outro fator relevante foi o indice de infecção natural do $T$. sordida pelo $T$. cruzi que foi maior do que o somatório de todos os outros índices juntos, das outras espécies sinantrópicas de triatomíneos.

Em Goiás, a segunda espécie sinantrópica de triatomíneo que deve merecer atenção da vigilância entomológica é $R$. neglectus, devido ter a mesma distribuição geográfica de $T$. sordida e ter a segunda maior infestação domiciliar (Figs. 2, 3).

\section{SUMMARY}

\section{Vectors' infestation of American tripanosomiasis in the domiciliary} environment in State of Goiás.

In the dwellings end annex triatomines of 9 species have been captured. The triatomines' ratios infestation in order based on how frequently they are found in dwellings: Triatoma sordida $-87.11 \%$, Rhodnius neglectus $-6.01 \%$, T.infestans $3.86 \%$, Panstrongylus megistus - 2.59\%, T.pseudomaculata - $0.24 \%$, P.diasi $0.12 \%$, T. costalimai - $0.08 \%$, P.geniculatus - $0.04 \%$, T.lenti $-0.03 \%$. The triatomines' natural infection ratios by Trypanosoma cruzi are $T$. sordida $-0.37 \%$,
SILVA, I.G.; SILVA, J.L.; CAMARGO, M.F.; ELIAS, C.N.; SANTOS, A.H.; SILVA, H.H.G.; ISAC, E.; ALVES, R.B.N. Infestação de vetores da tripanosomíase americana no ambiente domiciliar, no Estado de Goiás. Rev. Pat. Trop.24 ( 1 ): $41-47$, jan/jun. 1995

T.infestans $-0.11 \%$, P.megistus $-0.07 \%, T$. pseudomaculata $-0.02 \%$ and R.neglectu. $-0,01$.

KEYWORDS: American tripanosomiasis. Trypanosoma cruzi. Infestation. Triatomines. Vectors.

\section{REFERÊNCIAS BIBLIOGRÁFICAS}

1. EliAS, C.N.; SILVA, I.G. da; CAMARGO, M. de F. \& ALVES, R. de B. das N. Índice de infecção de triatomíneos pelo Trypanosoma cruzi, capturados no ambiente domiciliar no estado de Goiás em 1993. Rev.Pat.Trop., 23:169-174, 1994.

2. LUSTOSA, E. de S.; NAVES, H.A.M.; CARVALHO, M.E.S.D.;CALIL, F. \& MATOS, C.A. Distribuição geográfica de triatomíneos encontrados nos domicílios e peridomicílios nas micro-regiões do estado de Goiás. Rev.Goiana Med., 30:49-54, 1984.

3. SILVA, I. G. da. Nova técnica para leitura do xenodiagnóstico. Rev.Goiana Med., 36:35-39, 1990.

4. SILVA, I.G. da; SILVA, J.L. da; SILVA, H.H.G. da; CAMARGO, M. de F.; MOURA, A.F. de; ELIAS, M. \& SANTOS, A.H. dos. Distribuição dos vetores da tripanosomíase americana no ambiente domiciliar, no estado de Goiás, no período de 1984/ 88. An.Soc.ent. Brasil, 21:139-154, 1992.

5. SILVA,I.G. da; SILVA, H.G. da; SANTOS, V.L.V. dos; SILVA, J.L. da; CAMARGO, M. de F.; NAKANO, H.; SAHLA, L.A. \& ELIAS, M. Distribuição dos vetores da tripanosomíase americana capturados no ambiente domiciliar, no estado do Tocantins, no período de 1984/88. Rev.Pat.Trop., 20:165-171, 1991. 